\title{
2.2. ON THE SECULAR MOTION OF THE MEAN POLE
}

\author{
SHIGERU YUMi and YasuJiro WaKō \\ (International Latitude Observatory of Mizusawa, Japan)
}

\begin{abstract}
Analyzing the notable increases in the residual latitudes of the five ILS stations, local drifts of $-0 " .00156 /$ year for Mizusawa and of $+0 " .00105 /$ year for Ukiah were derived. Subtracting the apparent motion of the mean pole due to the local drifts, the secular motion of the mean pole is 0 ". 00220/year in the direction $77.7 \mathrm{~W}$ and this seems to be the real one.

\section{RÉSUMÉ}

En analysant les augmentations notables dans les latitudes résiduelles des cinq stations du SIL, on a déduit les dérives locales de -0 ".00156/an pour Mizusawa et +0 ". 00105/an pour Ukiah. Si on soustrait le mouvement apparent du pôle moyen qui est causé par les dérives locales, le mouvement séculaire du pôle moyen est 0 ". 00220/an dans la direction de $777^{\circ} 7 \mathrm{~W}$ et il semble être le mouvement réel.
\end{abstract}

Change of the position of the mean pole has hitherto been studied and discussed by many researchers, and the reality of secular change and libration were suggested by Sekiguchi (1), Hattori (2) and Markowitz (3) assuming that the relative positions of the ILS stations did not change with each other. However, there is a supposition that the real change in mean latitude of any station which is expected from a supposed displacement related with a crustal movement, or a change in the plumb line at the station, etc., would produce an apparent change of the mean pole.

The annual mean value of the residual latitudes at each station $(o-c)$, the difference between the observed latitude and the expected one computed from the derived polar coordinates, has a remarkable secular increase except for Carloforte in addition to a large fluctuation from year to year, as shown in Figures 1 and 2. See (4).

It amounts to $0.03-0$ ".07 for the latest years, and the probable error of the derived coordinates of the pole is about 0.03 , which far exceeds the value of a several thousandths of a second of arc expected from the precision of the monthly mean latitudes and the scattering of the monthly values of $(o-c)$. This suggests the reality of local errors or local secular drift accompanied by fluctuations at any station and the resulting apparent motion of the mean pole. In this paper, however, only the secular part is dealt with, because the available data and number of stations are considered to be too few to discuss the effect of fluctuation in location.

Analyzing the residual latitudes $(o-c)$ of the five ILS stations and comparing them with each other, the secular change in the local error at Mizusawa $\left(-0^{\prime \prime} .00312\right)$ or at 


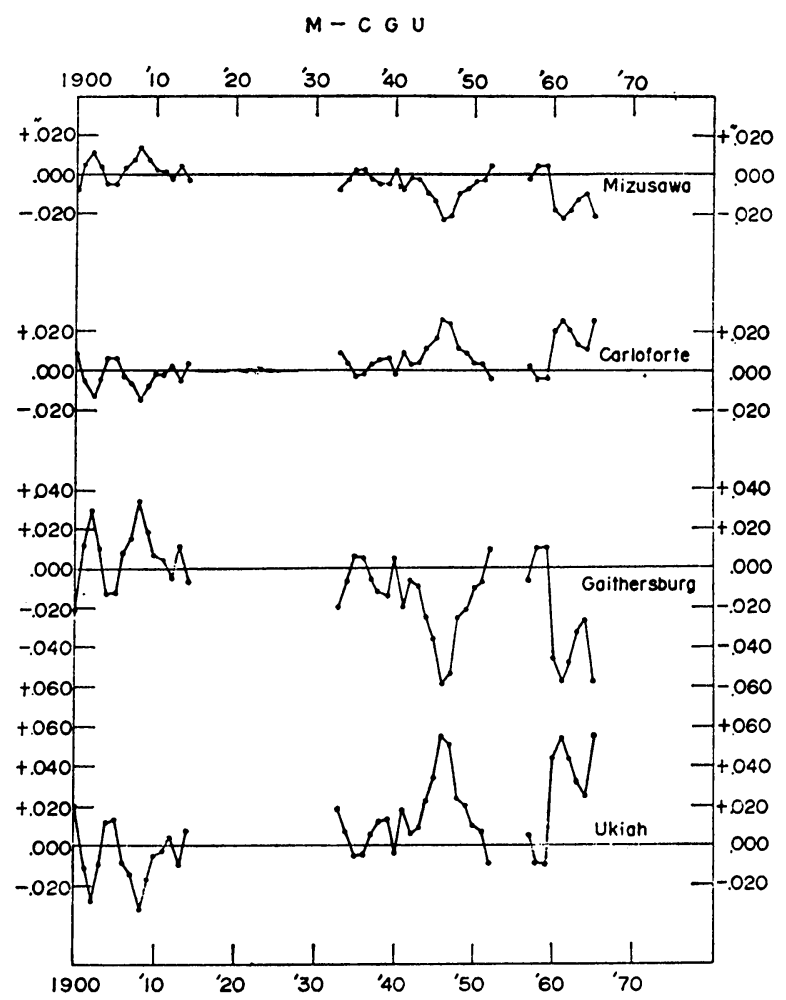

FIG. 1. Residuals, o-c, for 4 stations.

Ukiah ( +0.00209$)$ was found to be the main cause of the obtained secular change of $(o-c)$ at each station. Assuming the half and half secular changes, -0 0.00156/year for Mizusawa and +0 ".00105/year for Ukiah, the secular changes in the residual latitudes of all the ILS stations may almost vanish. This indicates the possibility of local drift at any station, the amount of which is supposed to be about 0 ".001/year.

This assumption leads to the conclusion that the secular change of the mean pole which has hitherto been obtained from the results of Mizusawa, Kitab, Carloforte, Gaithersburg and Ukiah is reduced to $\frac{1}{2}$ in its $x$-component and $\frac{3}{4}$ in the $y$-component. The resulting magnitude of the reduced secular change is

$$
\left.\begin{array}{l}
\Delta S_{x}=+0.00047 \\
\Delta S_{y}=+0.00215
\end{array}\right\} \quad \Delta S=0 " .00220, \quad \theta=77^{\circ} .7 \mathrm{~W}
$$

and this change seems to be real.

This assumption also leads to another conclusion, that the probable errors of the coordinates of the barycentres for the latest years which amount to about \pm 0 ".03 in 


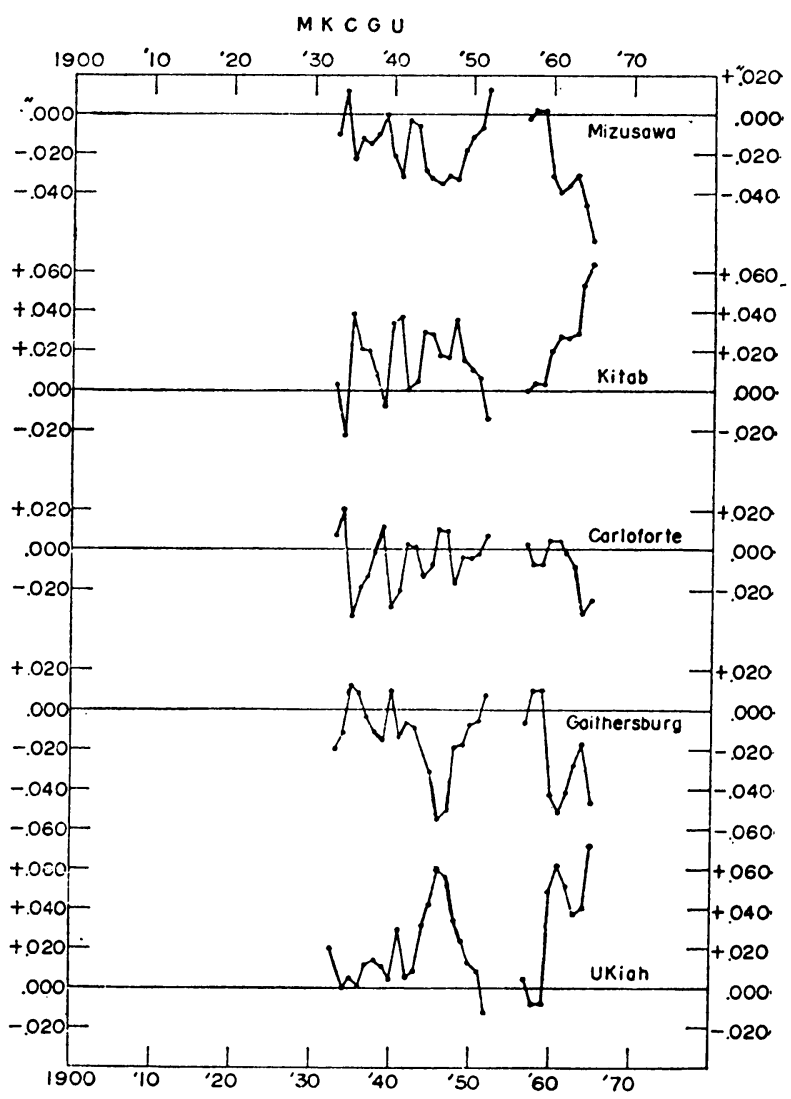

FIG. 2. Residuals for 5 stations.

the current system of MKCGU are reduced to a relatively small amount of \pm 0 ". 01 , even though this is not quite the satisfactory one.

The treatment of the residual latitudes of the stations also suggests the necessity of some corrections to the mean latitudes in the new system $1900-05$, that is +0 ".036 for Mizusawa and -0.026 for Ukiah.

In order to detect more precisely the reality of the local drifts of the stations and of the resulting secular motion of the mean pole, additional stations on the same parallel of $+39^{\circ} 8^{\prime}$ should be established to provide a nearly equal distribution in longitude. For the time being, Tientsin in China and Cincinnati in the U.S.A. are the stations desired. On the other hand, as the astronomical time and latitude are observed referring to the plumb line at the station on the earth crust, change of plumb line and the deformation of the earth crust which might be related with the continental drift should be observed geographically and geodetically in parallel with the astronomical observations. 
As far as the latitude is observed independently at any station after its own programme, the mean latitude of the independent station cannot be obtained precisely from the observed latitudes, even corrected by the chain method, but the error in the proper motion will apparently be introduced into the result as the secular variation of the local drift. The errors in declination and proper motion should be determined by meridian circle observations.

Detailed discussions and some comments are given in a recent paper by the present authors in (4).

\section{References}

1. Sekiguchi, N. (1954) Publ. astr. Soc. Japan, 5, $109 . \quad$ Sekiguchi, N. (1956) Publ. astr. Soc. Japan, 8, 13.

2. Hattori, T. (1959) Publ. int. Latit. Obs. Mizusawa, III, No. $1,1$.

3. Markowitz, Wm. (1960) Methods and Techniques in Geophysics, p. 350.

4. Yumi, S., Wakō, Y.(1966) Publ. int. Latit. Obs. Mizusawa, V, No. 2, 75-77. 